\title{
La apertura para la discusión \\ en clase y su relación con la \\ educación para la ciudadanía*
}

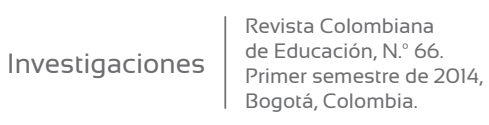

\author{
//The opening for discussion \\ in class and its relation to \\ citizenship education
}

\section{//A abertura à discussão \\ em aula e sua relação com a \\ educação para a cidadania}

Angélica Valencia Serna**

Harvy Vivas Pacheco***
Recibido: 03/02/2014

Evaluado: 17/03/2014 $09 / 04 / 2014$

\footnotetext{
* $\quad$ Este artículo es resultado de la investigación "La educación para la ciudadanía en Colombia: un análisis de las pruebas internacionales sobre educación cívica de la IEA", financiada por el Icfes en la convocatoria para estudiantes de posgrado de 2011, la cual culminó satisfactoriamente en 2013. La investigación se realizó en el marco del grupo de investigación Economía Regional y Ambiental (Gera), Línea de investigación Economía y Sociología de la Educación, Universidad del Valle, Cali, Colombia. Agradecemos los valiosos comentarios y sugerencias de los evaluadores anónimos designados por el comité editorial de la revista, los cuales contribuyeron a enriquecer la versión final de este artículo. Magíster en Sociología - Universidad del Valle, Cali, Colombia. Docente Universidad del Valle - sede Palmira. Correo electrónico: angelica.valencia.serna@correounivalle.edu.co / valseran27@gmail.com

Doctor en Economía Aplicada - Universitat Autònoma de Barcelona, Barcelona, España. Docente Universidad del Valle - Campus Universitario Meléndez - Facultad de Ciencias Sociales y económicas, Departamento de Economía. Correo electrónico: harvy.vivas@correounivalle.edu.co
}

\section{Resumen}

Este artículo analiza la relación existente entre la percepción que tienen los estudiantes colombianos de grado octavo (cuya edad aproximada es 14 años) acerca de la apertura para la discusión en clase propiciada por los docentes y el puntaje obtenido en conocimiento cívico. Además, el artículo estudia la relación entre la percepción de apertura en el aula y la participación efectiva de los estudiantes en actividades comunitarias (vinculación a organizaciones ambientales, juveniles, políticas, de derechos humanos, culturales, étnicas, grupos de voluntariado y organizaciones orientadas a causas sociales). El estudio utiliza los datos de 6.204 estudiantes colombianos incluidos en la muestra del International Civic and Citizenship Study (ICCS) de 2009. Inicialmente se realiza un análisis exploratorio con coeficientes de fiabilidad de Cronbach y luego se estiman los coeficientes de regresión estructural con recursos técnicos provenientes del path analysis. La hipótesis de partida es que la democratización de la escuela, en este caso específico, la participación propiciada por los docentes en el desarrollo de las clases, debe contribuir no solo a que los estudiantes obtengan mayores puntajes sino también a incrementar su participación social en la comunidad. Los resultados obtenidos en las estimaciones efectivamente confirman que la percepción de participación propiciada por los docentes en el salón de clase se relaciona directa y positivamente con los puntajes obtenidos por los estudiantes en las pruebas de conocimiento cívico, así como con la participación en actividades comunitarias. Finalmente, los coeficientes de regresión estimados mediante el path analysis resultaron estadísticamente significativos.

\section{Abstract}

This paper analyzes the relationship between Colombian 8th grade (aprox. 14 years old) students' perception about the openness to discussion in class,

\section{Palabras Clave}

Educación para la ciudadanía, apertura para la discusión en clase, participación efectiva, ICCS.

\section{Keywords}

Citizenship education, openness to classroom discussion, effective participation, ICCS.

\section{Palavras chave}

Educação para a cidadania, abertura para a discussão em aula, participação efetiva, ICCS. 
propitiated by teachers, and their score in citizenship knowledge tests. In addition, the article studies the relationship between the perception of openness in the classroom and the effective participation of students in community activities (enrolment in environmental, juvenile, political, human rights, cultural and ethnic organizations, groups of voluntary work and social projects enlistment). The study uses data of 6204 Colombian students included in the sample of the International Civic and Citizenship Study of 2009 (ICCS). An exploratory analysis of Cronbach coefficients of reliability was carried out and, then structural regression coefficients (path analysis) were estimated. The initial hypothesis is that democratization of school, in this specific case, participation propitiated by teachers in the classroom, contributes to students' high scores in tests evaluating citizenship related topics and increases their social participation in community activities. The results confirm that participation propitiated by teachers in the classroom relates directly to the citizenship test score obtained by students and to participation in community activities. Regression coefficients were statistically significant in this study.

\section{Resumo}

Este artigo analisa a relação entre a percepção dos estudantes de terceiro ano de ensino secundário (com idade de aproximadamente 14 anos) respeito da abertura à discussão em aula propiciada pelos professores e o escore obtido em conhecimento cívico. Igualmente, estuda a relação entre a percepção de abertura na aula e a participação efetiva dos estudantes em atividades comunitárias (vinculação a organizações ambientais, de jovens, políticas, de direitos humanos, culturais, étnicas, grupos de voluntariado e organizações destinadas a causas sociais). O estudo usou dados de 6204 estudantes colombianos incluídos na amostra do International Civic and Citizenship Study (ICCS) de 2009. Inicialmente se fez uma análise exploratória com coeficientes de fiabilidade de Cronbach e depois estimaram-se os coeficientes de regressão estrutural com recursos técnicos do path analysis. A hipótese inicial foi que a democratização da escola, neste caso específico, a participação propiciada pelos professores durante as aulas deve contribuir para que os estudantes obtenham escores mais altos e aumentem sua participação social na comunidade. Os resultados obtidos nas estimações confirmaram que a percepção de participação propiciada pelos professores na sala de aula tem relação direta e positiva com os escores obtidos pelos estudantes nas provas de conhecimento cívico, bem como com sua participação em atividades comunitárias. Finalmente os coeficientes de regressão estimados mediante o path analysis foram estatisticamente significativos.

Para la sociedad actual es imprescindible la educación de sujetos críticos y participativos, individuos que vayan más allá de la adquisición de destrezas para la realización de actividades productivas y que sean ciudadanos que participen activamente en los espacios políticos y comunitarios que la sociedad ofrece; sujetos que contribuyan a la consolidación de la democracia y a la cualificación de las demandas políticas y sociales.

Esta imperiosa necesidad no solo es sentida por Colombia, se trata también de una urgencia y preocupación de los países democráticos, por cuanto se entiende la participación como un elemento central en el desarrollo de la sociedad. Por todas estas 
razones, en los últimos años se han venido adelantando ingentes esfuerzos en la educación para la ciudadanía de los niños y jóvenes, lo que ha implicado nuevos retos tanto para la escuela como para la sociedad en general, como aparece consignado en diversos estudios disponibles en publicaciones nacionales e internacionales (Dewey, 1995; Durkheim, 1996 y 2002; Echavarría, 2003; Cox, Jaramillo y Reimers, 2005; García Roca, 2007; Unesco, 2008; RuizSilva y Chaux, 2005; Icfes, 2011).

Este estudio se interesa de manera particular en el rol que los docentes juegan en la educación para la ciudadanía de los niños y jóvenes. Es ampliamente conocida en el campo de la educación la importancia de las actividades pedagógicas desarrolladas en clase para el logro de los objetivos propuestos en las distintas áreas del conocimiento, aspecto que ha sido tratado por diversos autores, entre los que cabe destacar los trabajos de Darling-Hammond, 2000; Mayer et al., 2000; Losito y Mintrop, 2001; Torney-Purta et al., 2005a y 2005b; Akbari y Allvar, 2010; Alivernini y Manganelli, 2011 y León et al., 2012.

Ahora bien, dichas actividades en clase se enmarcan en lo que la literatura especializada denomina el clima, diferenciando el clima escolar, cuando el análisis se centra en la institución, del clima de aula, que se refiere específicamente al microespacio del salón de clases (Molina y Pérez, 2006). La literatura también señala que el clima puede desarroIlarse entre dos puntos extremos, por un lado, un clima cerrado y autoritario, desfavorable para el desarroIlo interpersonal de los estudiantes y negativo para la convivencia y el proceso de aprendizaje (Molina y Pérez, 2006). De otro lado, un clima abierto, más democrático, propicia la formación integral del estudiante (Molina y Pérez, 2006), pues este posibilita metodologías que favorecen la capacidad de escucha, respeto y tolerancia. Asimismo, otros autores como Mapiasse (2007) efectivamente recalcan que el clima democrático en el salón de clase contribuye no solo al desarrollo de habilidades cognitivas, sino también a la adquisición y proyección de destrezas sociales que se pueden expresar en la participación activa dentro y fuera de la institución educativa.

La hipótesis de partida del estudio aquí planteado es que la democratización de la escuela, específicamente lo concerniente a la participación facilitada por los docentes en el desarrollo de las clases, es decir, la apertura de estos para la discusión, debe contribuir no solo a que los estudiantes obtengan mayores puntajes sino también a incrementar su participación social en la comunidad. A partir de esta hipótesis, el trabajo estudia la relación entre el conocimiento cívico, de acuerdo con las escalas de medición de la prueba ICCS, y la percepción que tienen de los estudiantes acerca de la apertura para la discu- 
sión en clase propiciada por los docentes. Asimismo, se analiza la asociación entre dicha percepción y la participación efectiva de los estudiantes en actividades comunitarias, entre las que se resalta la vinculación a organizaciones ambientales, juveniles, políticas, de derechos humanos, culturales, étnicas, grupos de voluntariado y organizaciones orientadas a causas sociales.

La investigación utiliza los datos del International Civic and Citizenship Study (ICCS) de 2009, realizado por la International Association for the Evaluation of Educational Achievement (IEA). En el ICCS participaron 38 países y su objetivo era conocer qué tan bien preparados están los jóvenes para asumir su rol como ciudadanos de una sociedad moderna, donde los cambios ocurren con mucha rapidez (Schulz et al., 2008). El tamaño muestral para Colombia incluyó 196 escuelas, 2.010 docentes y 6.204 estudiantes.

Los resultados para Colombia en el ICCS la ubican en el puesto 26 -en un ranking de 36 países ${ }^{1}$ - con un puntaje de 462 puntos (con una media internacional fijada en 500) y una desviación estándar de 81 (fijada en 100 para la prueba). Para la clasificación de los participantes según los resultados obtenidos, se diseñó una escala de conocimiento que constó finalmente de cuatro niveles de desempeño: por debajo del nivel 1, que agrega a aquellos estudiantes que obtuvieron puntajes inferiores a 395 puntos; nivel 1, estudiantes que lograron entre 395 y 478 puntos; nivel 2, estudiantes con puntajes entre 479 y 562 puntos; nivel 3, estudiantes que alcanzaron 563 puntos o más ${ }^{2}$ (Schulz, 2010).

Es importante precisar que el ICCS evaluó tanto conocimientos en educación cívica como percepciones cívicas y ciudadanas, valores y actitudes de los estudiantes con edad aproximada a los 14 años $^{3}$. El modelo teórico del ICCS (figura 1) considera tanto los aspectos individuales como los sociales que están implicados en la educación para la ciudadanía de los estudian-

1 Dos de los participantes, Países Bajos y Hong Kong, no cumplieron con la totalidad de requisitos para ser parte de las comparaciones internacionales.

2 Estos últimos son estudiantes con mayores habilidades, destrezas y conocimientos en los diferentes dominios que evalúa el ICCS, los cuales argumentan con solidez sobre temas políticos y sociales, además de establecer relaciones entre la organización política y social y la institucionalidad. Los del nivel 2 manejan conceptos de educación para la ciudadanía y reconocen cómo funcionan las leyes e instituciones, pero no alcanzan razonamientos tan elaborados como los del nivel 3. Los del nivel 1 tienen conocimientos elementales de conceptos básicos de educación para la ciudadanía y los que se ubican por debajo de este nivel de desempeño no alcanzan los ámbitos elementales de comprensión de la educación cívica.

3 Para el caso de Colombia, pertenecientes al grado octavo. 
tes y asume que estos últimos se encuentran en el centro de este proceso, rodeados de valores, prácticas y del discurso público.

Es a través de un conjunto de portadores (la familia, la escuela, los pares, entre otros) que estos elementos logran influenciar a los estudiantes. Es claro, entonces, que el ICCS no limita la educación para la ciudadanía a la escuela, pues las distintas variables que proporciona la base de datos permitirían hacer una aproximación a esos agentes portadores y su importancia en la educación para la ciudadanía, pero en este trabajo se hace énfasis en la influencia de la escuela, particularmente en el rol de los docentes a partir de la percepción que tienen los estudiantes de la apertura para la discusión en clase que estos propician.

Figura 1. Modelo teórico del estudio sobre educación cívica de la IEA.
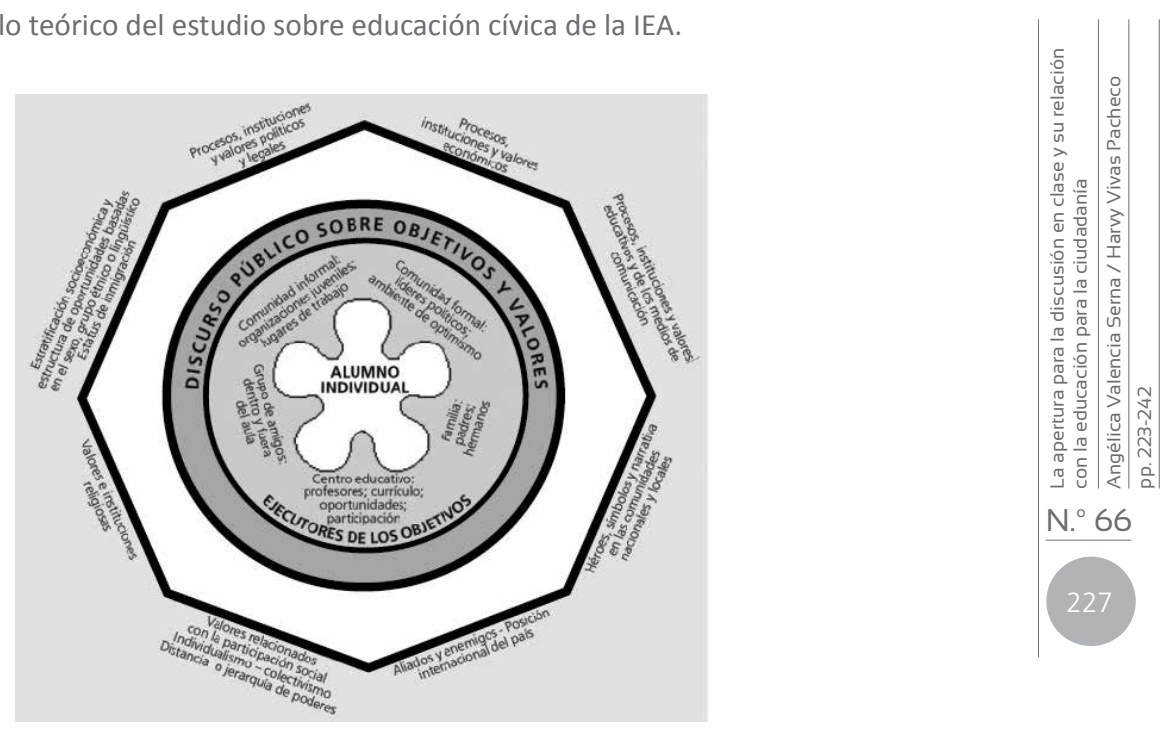

Fuente: Schulz et al., 2008.

En este sentido, es preciso mencionar que teóricamente se espera que mayores opciones de participación en clase se vean reflejadas en mejores puntajes por parte de los estudiantes, y estas mayores opciones están intrínsecamente ligadas al clima democrático que se pueda propiciar en el aula de clase, como se expuso antes. Una premisa asociada a lo anterior es la que soporta la idea de la democratización escolar, como estrategia reciente, pero también de la educación para la ciudadanía ${ }^{4}$, pues se espera con ello generar un aprendizaje significativo en los estudiantes en lo que concierne

4 Es importante tener en cuenta en este momento que educación para la ciudadanía es solo una de las múltiples denominaciones que se da a la educación con objetivos similares, pues existen otras que pueden tener algunas diferencias conceptuales, entre ellas, educación cívica (más genérica y la que adopta la IEA), educación política, educación para la democracia, educación para la paz, formación ciudadana, entre otras. 
a la participación política y social, así como en la toma de decisiones, es decir, preparar a los estudiantes para su participación política en la vida adulta (Unesco, 1996; Gil Villa, 1997; Guttman, 2001; Álvarez, 2005; Orellana y Minte, 2011).

En otras palabras, la democratización de la escuela en todos sus aspectos se soporta en la intención de favorecer la participación de los estudiantes en el presente, pero también en la necesidad de prepararlos para el ejercicio de una ciudadanía activa y comprometida con el bien común en el futuro. En Colombia se han desarrollado distintos trabajos orientados a tratar las implicaciones de esta concepción, entre ellos se encuentran los estudios de Álvarez et al., 2002; Echavarría, 2003; Álvarez, 2005; Pinilla y Torres, 2006, entre otros. Cox, Jaramillo y Reimers (2005) Ilaman la atención a tener en cuenta que no solo se deben pensar las prácticas pedagógicas y la organización de la escuela, sino también el desarrollo de competencias en una idea más integral de la educación para la ciudadanía.

Estos mismos autores señalan que para el contexto latinoamericano es central la discusión del papel del docente en este proceso, aún más si se considera que en esta sección del continente es reconocido que estos son el eslabón más débil en las reformas educativas (Cox, Jaramillo y Reimers, 2005), en particular para lo que concierne a la educación para la ciudadanía. Los trabajos de Torney-Purta et al. (2005a y 2005b) y Alivernini y Manganelli (2011) también se han interesado en estas mismas preocupaciones y retos.

De esta manera, reconociendo la importancia teórica de un clima democrático en clase, su pertinencia en la educación para la ciudadanía y el rol que los docentes juegan en todo ello, se ha considerado de interés analizar la percepción que tienen los estudiantes acerca de la apertura para la discusión en clase propiciada por los docentes en relación, por un lado, con el puntaje obtenido en conocimiento cívico y, por otro, con la participación efectiva en actividades comunitarias, utilizando para ello los resultados del ICCS.

\section{Datos y metodología}

La base estadística de este estudio corresponde a la muestra extraída para Colombia por el ICCS en 2009, que se aplicó a 6.204 estudiantes distribuidos en 196 planteles educativos públi- 
cos y privados de todo el país ${ }^{5}$. De acuerdo con el Informe de Resultados de Colombia (Icfes, 2011), la diferencia entre el desempeño de hombres y mujeres no es estadísticamente significativa; por el contrario, las diferencias observadas entre instituciones públicas y privadas sí lo son a favor de las segundas. Asimismo, este informe señala que los estudiantes de un nivel socioeconómico más alto obtuvieron mejores puntajes, situación que se ha corroborado en análisis previos realizados utilizando los resultados obtenidos en otras pruebas estandarizadas.

La base de microdatos del ICCS está conformada por 283 variables a partir de un conjunto de las cuales el ICCS construyó 24 escalas que permiten indagar aspectos más complejos, enriqueciendo el margen de análisis que la prueba permite. Además de las escalas señaladas para este estudio, pueden encontrarse la de percepción de la importancia de la ciudadanía como movimiento social, la confianza de los estudiantes en las instituciones cívicas, el apoyo que ofrecen a los valores democráticos, sus actitudes hacia la igualdad de género, entre otras.

En la dimensión cognitiva la base estadística cuenta con valores plausibles que resumen los conocimientos, capacidades y destrezas de los estudiantes de acuerdo con sus respuestas y valoraciones en cada uno de los ítems aplicados. De acuerdo

5 La IEA puso a disposición del público la base de datos del ICCS en 2011. con la teoría de respuesta al ítem (TRI) con información incompleta y un conjunto de variables de condicionamiento o de contexto se obtienen distribuciones que condensan los puntajes probables de los participantes ${ }^{6}$.

Estos valores permiten la estimación de parámetros poblacionales con un mayor grado de consistencia que la obtenida con estimaciones puntuales, en la medida que se elaboran con procedimientos de imputación que consideran de manera simultánea las respuestas individuales a los dominios asignados y las ajusta con valores específicos asociados al contexto escolar, familiar y local de los estudiantes. Como es usual en las pruebas estándar internacionales, en este caso, la base de microdatos del ICCS arroja cinco valores plausibles que provienen de la función de respuesta a posteriori de cada alumno.

6 De acuerdo con las metodologías de competencias estándar aplicadas por la IEA en sus diferentes pruebas, cada estudiante responde una parte del cuestionario y un número parcial de ítems, de tal manera que, mediante procedimientos de imputación se obtienen los valores plausibles que provienen de la predicción del comportamiento en el total de los ítems, según los condicionamientos de cada estudiante, el cual depende de un conjunto de variables de contexto. De acuerdo con la Teoría de Respuesta al Ítem (TRI), se espera que los estudiantes con habilidades descollantes en un grupo respondan preguntas fáciles con acierto, mientras que los que no cuentan con las habilidades necesarias fallen en las preguntas difíciles. Por tal razón, a diferencia del modelo clásico de medida, la probabilidad de respuesta acertada se asocia al grado de dificultad y a la habilidad del que responde. Ver Diseño muestral de la prueba del ICCS e implicaciones para el análisis, Bogotá, 7 y 9 de abril de 2010, IEADPC Research and Analysis Unit (diapositivas). 
Además de los valores plausibles, la prueba ICCS cuenta con un conjunto de constructos o escalas que condensan información compleja relacionada con las actitudes y percepciones de los estudiantes y docentes acerca de la participación ciudadana, la apertura democrática en el salón de clase, la participación en las instancias de gobierno de las escuelas, así como en organizaciones ambientales, políticas y sociales por fuera del plantel, entre otros. Esto constructos se obtienen a partir de un conjunto de indicadores que van cargados de contenido informacional relevante sobre las actitudes de los estudiantes y docentes y que resulta decisiva para su medición. Como se mencionó antes, el ICCS cuenta con 24 de estas escalas obtenidas mediante estimaciones ponderadas de probabilidad, WLE, de manera que cada una de ellas tiene una media internacional de 50 y una desviación estándar de 10 para efectos de comparación.

Además de los puntajes nacionales en las pruebas de conocimiento cívico ${ }^{7}$, en este artículo se utiliza la escala de percepción sobre apertura de los docentes para la discusión en el salón de clase, así como la de participación efectiva de los estudiantes en actividades comunitarias.

Estos constructos se evaluaron para el caso específico colombiano mediante pruebas de fiabilidad estadística con el coeficiente alpha de Cronbach (a), el cual permite evaluar la consistencia interna de las escalas (no observables) a partir de los indicadores (observables) que configuran el modelo de medida sugerido por el ICCS. Este coeficiente toma valores entre cero y uno, de manera que valores altos proporcionan indicios de consistencia, mientras que valores por debajo de 0,5 indican que los ítems que conforman la escala no son fiables en su configuración.

La especificación estadística del coeficiente alpha de Cronbach (expresión 1) tiene en cuenta el número de ítems de cada una de las escalas o subescalas, la varianza de los ítems y la varianza de la prueba total, incluyendo así la longitud de la prueba (número de ítems) y la proporción de la varianza total de la prueba atribuible a la covarianza entre sus elementos (Valencia, 2013 y Valencia y Vivas, en prensa): 


$$
\alpha=\frac{N}{N-1}\left[1-\frac{\sum_{i=1}^{n} S_{i}^{2}}{S_{\text {sum }}^{2}}\right]
$$

En donde:

$\mathrm{N}$ : número de ítems considerados en cada escala

$\mathrm{Si}^{2}$ : suma de varianzas de cada ítem

$\mathrm{S}^{2}$ sum: varianza de la prueba como conjunto (escalas o subescalas)

Finalmente, en este apartado metodológico es preciso anotar que la contrastación de las hipótesis de este estudio utiliza recursos estadísticos de path analysis. Estos procedimientos, que con frecuencia se observan en el ámbito estadístico de los modelos de ecuaciones estructurales, exploran o confirman relaciones entre variables y plantean de manera explícita la presencia de relaciones lógicas sustentadas en enfoques teóricos.

De manera consistente con los planteamientos señalados previamente, el modelo planteado establece que la percepción de los estudiantes sobre la apertura de los docentes para la discusión en el salón de clase se relaciona positivamente con el resultado de las pruebas de conocimiento cívico (medido en la escala nacional de ICCS) y a la vez se asocia positivamente con la participación efectiva de los estudiantes en actividades comunitarias. El puntaje se modela con un efecto de contexto que se expresa en el índice socio-económico disponible en la base de microdatos (figura 2).

Figura 2. Colombia: path analysis.

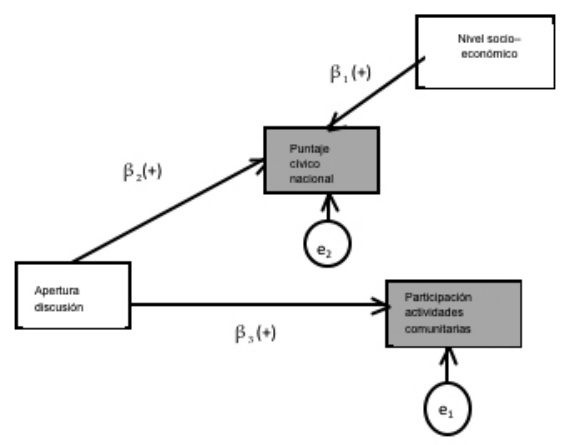

* Los rectángulos contienen las variables endógenas y exógenas del modelo y los óvalos, los errores, entre paréntesis las relaciones esperadas.

Los betas $\left(b_{\mathrm{j}}\right)$ corresponden a los pesos de regresión y entre paréntesis aparecen los signos esperados. El path analysis planteado corresponde a un modelo recursivo en la medida que no considera la presencia de causalidades recíprocas ni correlaciones entre los errores $\left(\mathrm{e}_{\mathrm{j}}\right)$, como es el caso de los denominados modelos no recursivos. De acuerdo con esta estructura, el puntaje y la participación comunitaria operan como las variables endó- 
genas del modelo, mientras que la percepción de apertura y el índice socio-económico, como variables exógenas. Los errores miden la variabilidad no explicada por las variables exógenas. Las estimaciones de los pesos de regresión y de los demás parámetros se realizan mediante máxima verosimilitud.

A diferencia de los modelos de regresión uniecuacionales, el path planteado permite estudiar la relación simultánea entre las variables y obtener, además del efecto total, los efectos directos e indirectos, si es el caso. La expresión 2 muestra la estructura matricial del modelo cuyos resultados se presentan al final de la siguiente sección.

Expresión 2.

$$
\left[\begin{array}{c}
\text { Puntajeciviconacional } \\
\text { Participacióncomunitaria }
\end{array}\right]=\left[\begin{array}{cc}
\beta_{2} & \beta_{1} \\
\beta_{3} & 0
\end{array}\right] *\left[\begin{array}{c}
\text { Aperturadiscusión } \\
\text { Nivelsocioeconómico }
\end{array}\right]+\left[\begin{array}{c}
e_{2} \\
e_{1}
\end{array}\right]
$$

\section{Análisis y discusión}

A partir de las estrategias metodológicas descritas anteriormente, el presente apartado presenta los resultados de las estimaciones. En primer lugar, se hace mención a los resultados de consistencia usando el coeficiente alpha de Cronbach $(\alpha)$, prosiguiendo con el análisis de los resultados de la asociación entre la percepción de la apertura de los docentes para la discusión en clase y el puntaje obtenido por los estudiantes $y$, finalmente, se presentan los hallazgos asociados a la relación entre la percepción de la apertura para la discusión en clase y la participación de los estudiantes en actividades comunitarias.

\section{Fiabilidad de las escalas}

A continuación se presentan los resultados de las estimaciones del coeficiente alpha de Cronbach (tabla 1) para las escalas de interés en este artículo, percepción de la apertura para la discusión en clase y participación en actividades comunitarias, y se detallan los indicadores que las conforman. Para la primera de ellas, se indagó por la estimulación que propician los docentes para la toma de decisiones y expresar las opiniones, así como para proponer y discutir temas sociales y políticos. Para la segunda, participación en actividades comunitarias, se indagó por la vinculación de los estudiantes a instituciones políticas, 
Tabla 1. Estimaciones del coeficiente alpha de Cronbach ( $\alpha$ ).

\begin{tabular}{|c|c|c|c|c|}
\hline \multicolumn{5}{|c|}{ Percepción sobre la apertura para discutir en clase } \\
\hline Escala & Etiqueta escala & $\alpha$ & Indicadores & $\begin{array}{l}\alpha \text { si se elimina } \\
\text { el elemento }\end{array}$ \\
\hline \multirow{6}{*}{$\begin{array}{l}\text { Cuando se discuten } \\
\text { asuntos políticos y } \\
\text { sociales en las cla- } \\
\text { ses regulares, ¿cada } \\
\text { cuánto ocurren las } \\
\text { siguientes cosas? }\end{array}$} & \multirow{6}{*}{$\begin{array}{l}\text { Apertura discu- } \\
\text { sión }\end{array}$} & \multirow{6}{*}{0,65} & $\begin{array}{l}\text { Los profesores estimulan a los } \\
\text { estudiantes a decidirse. }\end{array}$ & 0,60 \\
\hline & & & $\begin{array}{l}\text { Los profesores estimulan a los } \\
\text { estudiantes a expresar sus opi- } \\
\text { niones. }\end{array}$ & 0,58 \\
\hline & & & $\begin{array}{l}\text { Los estudiantes proponen temas } \\
\text { políticos actuales para discusión } \\
\text { en clase. }\end{array}$ & 0,64 \\
\hline & & & $\begin{array}{l}\text { Los estudiantes expresan opinio- } \\
\text { nes en clase, aunque éstas sean } \\
\text { diferentes de la mayoría. }\end{array}$ & 0,61 \\
\hline & & & $\begin{array}{l}\text { Los profesores estimulan a los } \\
\text { estudiantes a discutir los temas } \\
\text { con personas que tengan opinio- } \\
\text { nes diferentes. }\end{array}$ & 0,57 \\
\hline & & & $\begin{array}{l}\text { Los profesores presentan diferen- } \\
\text { tes puntos de vista de los temas } \\
\text { cuando los explican en clase. }\end{array}$ & 0,62 \\
\hline \multicolumn{5}{|c|}{ Participación cívica en actividades comunitarias } \\
\hline \multirow{7}{*}{$\begin{array}{l}\text { ¿Alguna vez ha } \\
\text { estado involucrado } \\
\text { en actividades de } \\
\text { cualquiera de las } \\
\text { siguientes organi- } \\
\text { zaciones, clubes o } \\
\text { grupos? }\end{array}$} & \multirow{7}{*}{$\begin{array}{l}\text { Participación } \\
\text { actividades } \\
\text { comunitarias }\end{array}$} & \multirow{7}{*}{0,71} & $\begin{array}{c}\text { Organización juvenil afiliada a } \\
\text { algún sindicato o partido polí- } \\
\text { tico. }\end{array}$ & 0,71 \\
\hline & & & Organización ambiental. & 0,67 \\
\hline & & & $\begin{array}{l}\text { Organización de derechos } \\
\text { humanos. }\end{array}$ & 0,66 \\
\hline & & & $\begin{array}{c}\text { Un grupo voluntario que haga } \\
\text { algo para ayudar a la comuni- } \\
\text { dad. }\end{array}$ & 0,66 \\
\hline & & & $\begin{array}{l}\text { Una organización que recolecte } \\
\text { dinero para una causa social. }\end{array}$ & 0,68 \\
\hline & & & $\begin{array}{l}\text { Una organización cultural étnica } \\
\text { (afrocolombianos, indígenas). }\end{array}$ & 0,70 \\
\hline & & & $\begin{array}{l}\text { Un grupo de gente joven en } \\
\text { campaña por un tema. }\end{array}$ & 0,68 \\
\hline
\end{tabular}

ambientales o culturales, entre las que se precisó en aquellas relacionadas con la defensa de los derechos humanos y los grupos de voluntariado.

En ambos casos, las estimaciones del coeficiente alpha de Cronbach son aceptables $^{8}$, sugiriendo que las dos escalas son consistentes internamente y

8 Aunque no existe consenso sobre los umbrales de este indicador de fiabilidad, para algunos autores los valores en el rango de 0,70 y 0,80 son suficientemente buenos en análisis exploratorio, valores inferiores a 0,60 son inaceptables y entre 0,65 y 0,70, aceptables; en el rango 0,70-0,80, respetables, y entre $0,80-0,90$, muy buenos. Los valores superiores a 0,90 sugieren redundancia de algunos ítems. 
que los ítems que las conforman efectivamente contienen información sobre el constructo. Estos hallazgos se refuerzan al observar los impactos marginales cuando se elimina cualquiera de sus indicadores, de tal manera que en ambas escalas dicho coeficiente se mantiene relativamente estable. Como se pudo apreciar (tabla 1), el coeficiente alpha de Cronbach para la escala percepción de la apertura para la discusión en clase alcanza un valor de 0,65 y para la de participación en actividades comunitarias llega a 0,71 , valores que resultaron aceptables. En el caso de la percepción de la apertura para la discusión en clase, se resalta la importancia de los indicadores relacionados con el estímulo a expresar opiniones y a discutirlas con personas con diferentes puntos de vista, pues al ser eliminado alguno de estos dos elementos el coeficiente alpha de Cronbach se afectaría más que en el caso de los otros indicadores.

\section{Apertura de la discusión en clase y puntaje cívico nacional}

Como se mencionó antes, la escala de percepción de la apertura de los docentes para la discusión en clase se construyó a partir de las respuestas de los estudiantes a la pregunta orientada a indagar por cada cuánto ocurrían algunas experiencias asociadas a la participación y al estímulo de la discusión de temas políticos y sociales (tabla 1). Esta escala tiene una media internacional de 50 puntos y Colombia alcanzó un puntaje de 50,14.

De acuerdo con lo esperado teóricamente según algunos estudios reseñados en el primer apartado de este documento, los resultados evidencian que a un mayor rango de percepción de la apertura de los docentes para la discusión en clase, los estudiantes obtuvieron mayor puntaje. A continuación se presentan los resultados (tabla 2) para el caso colombiano, donde se muestra el incremento en la puntuación obtenida por los estudiantes a medida que aumenta su grado de percepción de la apertura de sus docentes.

En el campo de la pedagogía y de la educación es ampliamente tratado lo relacionado con la necesidad de generar la participación en clase como mecanismo para el logro de los objetivos propuestos en las distintas áreas, pero también como medio de preparación para la vida social y política en la adultez, como se precisó en el primer apartado. Estos resultados permitirían 
corroborar estos planteamientos, tal como además lo presenta el caso de Dinamarca, otro participante en el estudio, que quedó segundo en el ranking de países participantes (con 576 puntos) y quien obtuvo el mayor puntaje $(54,72)$ en esta escala; pero no así el caso de Corea (del Sur), tercero en el ranking internacional (565 puntos), pero que obtuvo el puntaje más bajo en esta escala $(38,12)$, por lo que profundizar más en estos hallazgos podría ser enriquecedor para la comprensión de las estrategias didácticas que se utilizan y sus resultados en relación con los objetivos propuestos; sin embargo, dicho análisis escapa a los alcances de este artículo.

Tabla 2. Apertura de los docentes para la discusión en clase y logros de los estudiantes.

\begin{tabular}{|c|c|c|c|c|}
\hline País & $\begin{array}{c}\text { Rangos } \\
\text { apertura* }\end{array}$ & \% & Puntaje** & $\begin{array}{c}\text { Desviación } \\
\text { estándar }\end{array}$ \\
\hline & & & & \\
\hline & $<42,92$ & 15,83 & 423,36 & 73,17 \\
\hline Colombia & {$[42,92-48,85)$} & 25,62 & 448,79 & 78,62 \\
& {$[48,85-59,29)$} & 41,79 & 475,75 & 77,48 \\
& $>59,29$ & 16,76 & 498,11 & 73,00 \\
\hline
\end{tabular}

Fuente: Vivas y Valencia (2011), a partir de la base de datos del ICCS/IEA, 2009

*El grado de apertura en el salón de clase, desde la perspectiva de los estudiantes, se expresa en rangos a partir de la variable original de la base de datos de los estudiantes. La escala utiliza como valores de referencia una media de 50 y una desviación estándar de 10.

**Los puntajes se obtienen utilizando los cinco valores plausibles de la dimensión cognitiva de la prueba y utilizan los ponderadores de la muestra de estudiantes.

\section{Apertura para la discusión en clase y participación comunitaria}

De otra parte, al considerar lo expuesto en el primer apartado, respecto a la relación teóricamente esperada entre participación en clase y en actividades comunitarias, la correlación de Pearson da cuenta de dicha relación, es decir, efectivamente existe una asociación positiva entre la apertura para la discusión en clase que los estudiantes perciben y su participación en actividades comunitarias (tabla 3).

Esta misma situación puede verse en otros países de la región (tabla 4), en los cuales se observa un comportamiento similar al de Colombia (que obtuvo 55,24 puntos en la escala de participación en actividades comunitarias) en cuanto a que obtuvieron puntajes por encima del promedio (50) en ambas escalas, como Guatemala $(57,69)$, México $(52,85)$ y Chile $(51,12)$. Nuevamente resalta el caso de Corea, que también obtuvo el menor puntaje en esta escala $(42,46)$, pero que en este caso no pone en discusión la hipótesis sino que contribuye a reafirmarla. 
República Dominicana y Paraguay, para el caso latinoamericano, son ejemplos de lo contrario, ya que obtuvieron una puntuación por debajo del promedio en la escala de percepción de la apertura para la discusión en clase, pero altos puntajes en la participación en actividades comunitarias, pues en el caso de República Dominicana obtuvo el mayor puntaje en esta última $(59,62)$.

Tabla 3. Correlaciones de Pearson.

\begin{tabular}{|c|c|c|}
\hline \multicolumn{3}{|c|}{ Correlaciones de Pearson (CP) } \\
\hline & & $\begin{array}{l}\text { Percepción de la apertura para } \\
\text { la discusión en clase }\end{array}$ \\
\hline Puntaje cívico nacional & $\begin{array}{l}\mathrm{CP} \\
\mathrm{N}\end{array}$ & $\begin{array}{c}0,271 \\
640932\end{array}$ \\
\hline Participación actividades comunitarias & $\begin{array}{l}\mathrm{CP} \\
\mathrm{N}\end{array}$ & $\begin{array}{c}0,116 \\
638552\end{array}$ \\
\hline
\end{tabular}

Tabla 4. Resultados de las escalas de apertura para la discusión en clase y participación en actividades comunitarias para los países de América Latina incluidos en el ICCS.

\begin{tabular}{|c|c|c|c|}
\hline País & $\begin{array}{c}\text { Percepción de la } \\
\text { apertura para la dis- } \\
\text { cusión en clase }\end{array}$ & $\begin{array}{c}\text { Participación en acti- } \\
\text { vidades comunitarias }\end{array}$ & Puntaje ICCS \\
\hline Chile & 52,32 & 51,12 & 483 \\
\hline Colombia & 50,14 & 55,24 & 462 \\
\hline República Dominicana & 47,34 & 59,62 & 380 \\
\hline Guatemala & 52,61 & 57,69 & 435 \\
\hline México & 50,12 & 52,85 & 452 \\
\hline Paraguay & 49,45 & 57,10 & 424 \\
\hline Total global* & 50,94 & 52,06 & \\
\hline
\end{tabular}

**Incluye los 36 países de la muestra. Estimaciones con la muestra ponderada.

\section{Estimaciones path analysis}

En relación con las estimaciones con procedimientos path analysis, los ejercicios de contrastación de las hipótesis de comportamiento previamente señaladas efectivamente muestran que la percepción revelada por los estudiantes acerca de la apertura de los docentes en el salón de clase se relaciona positivamente con la escala de puntaje cívico nacional. La estimación por máxima verosimilitud del peso de regresión resultó estadísticamente significativa, alcanzando un valor estandarizado de 0,25. 
De manera similar, se pudo corroborar que esta percepción de apertura guarda una relación positiva con la participación efectiva de los estudiantes en actividades comunitarias, obteniendo un coeficiente estandarizado de 0,11 . El efecto de contexto, medido a través del índice socio-económico fue de 0,22 , lo cual resulta consistente con estudios previos y que reafirma la importancia que tienen las condiciones del hogar en los resultados de las pruebas de competencias.

La mirada general al conjunto de resultados sugiere que, como se ha podido apreciar en diversos estudios aplicados en países europeos y asiáticos, el clima democrático en el salón de clase aporta a las habilidades cognitivas y al desarrollo de la participación activa de los estudiantes en actividades comunitarias. La hipótesis de que la democratización de la escuela -medida de manera parcial a través de una mayor participación facilitada por los docentes en las clases- aporta de manera simultánea a las competencias cognitivas y coadyuva a la participación social en la comunidad, queda en principio contrastada satisfactoriamente para Colombia en el ejercicio propuesto en este artículo.

Figura 3. Colombia: pesos estandarizados de regresión del path analysis.

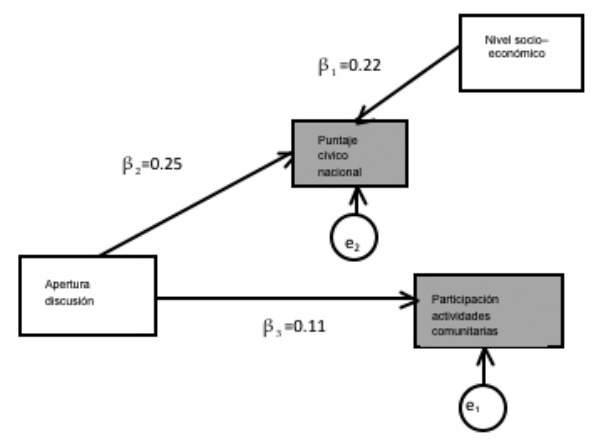

* Estimaciones por el método de máxima verosimilitud en Amos/SPSS.

No obstante, como es usual en este tipo de ejercicios estadísticos, los hallazgos se deben abordar con la debida reserva que exige el tratamiento riguroso de los datos cuando se introducen variables de control o se modela de manera explícita con el conjunto de ítems que configuran cada uno de los constructos estudiados. Al controlar por variables como el género o subgrupos socio-económicos, los resultados no presentaron diferencias significativas, por tal razón solamente se presentan los resultados estandarizados de las estimaciones (figura 3) y se resumen los valores no estandarizados y sus significancias estadísticas (tabla 5). 
Tabla 5. Colombia: pesos parciales de regresión del path analysis.

\begin{tabular}{l|l|l|l|l|l|}
\hline & Estimaciones(a) & S.E(b) & C.R(c) & P & Estandarizados \\
\hline $\begin{array}{l}\text { Participación actividades } \\
\text { comunitarias } \leftarrow \text { apertura } \\
\text { discusión }\end{array}$ & 0,120 & 0,014 & 8,421 & $* * *$ & 0,108 \\
\hline $\begin{array}{l}\text { Puntaje cívico nacional } \leftarrow \\
\text { nivel socio-económico }\end{array}$ & 2,201 & 0,120 & 18,303 & $* * *$ & 0,220 \\
\hline $\begin{array}{l}\text { Puntaje cívico nacional } \leftarrow \\
\text { apertura discusión }\end{array}$ & 0,284 & 0,014 & 20,576 & $* * *$ & 0,250 \\
\hline * Estimaciones en Amos/SPS. & & & & \\
\hline $\begin{array}{l}\text { (a) Pesos de regresión no estandarizados. } \\
\text { (b) S.E: errores estándar. }\end{array}$ \\
$\begin{array}{l}\text { (c) valores críticos. } \\
\text { (***) Significativo al } 1 \% .\end{array}$
\end{tabular}

\section{Conclusiones}

El estudio se orientó al análisis de la asociación de los resultados obtenidos por los estudiantes colombianos en el ICCS y su percepción de la apertura de los docentes para la discusión en clase. Asimismo, entre esta última y la participación efectiva de los estudiantes en actividades comunitarias. Para ello se estimó un modelo recursivo mediante procedimientos de path analysis que permite contrastar las relaciones simultáneas entre las variables pertinentes.

Los resultados de las estimaciones muestran que para el caso colombiano efectivamente existe una asociación positiva entre la apertura para la discusión en clase que generan los docentes y una mejor posición en la escala de desempeño establecida para el ICCS. Los resultados de las estimaciones muestran que a menor rango de percepción de apertura para la discusión en clase los estudiantes obtuvieron puntajes inferiores y la estimación por máxima verosimilitud del peso de regresión alcanzó un valor estandarizado, estadísticamente significativo.

Estos resultados respaldan los postulados teóricos en relación con la pertinencia de un clima democrático en la escuela y en particular en el aula de clase, toda vez que este propicia un ambiente donde más que enseñar conocimientos se posibilita una formación integral del estudiante. Esta formación integral apela a sujetos críticos, con capacidad de indagación y participación, con formación en valores como el respeto y la tolerancia, entre otros aspectos que son de suma importancia en la educación para la ciudadanía.

De igual forma, se corroboró la hipótesis de que la participación en clase puede promover una mayor participación en las actividades comu- 
nitarias. La estimación de la correlación de Pearson muestra una asociación positiva entre ambas escalas. Este hallazgo sugiere que efectivamente un clima democrático en clase brinda a los estudiantes las herramientas y motivación necesarias para su participación en actividades comunitarias.

Lo anterior aporta evidencia relevante de la pertinencia en nuestro contexto de actividades pedagógicas encaminadas a propiciar una mayor participación en el desarrollo de las clases, tanto para un mejor desempeño académico como por la relación positiva entre estas y la participación en actividades comunitarias, que, de acuerdo con los estudios previos presentados en el primer apartado, son elemento central del desarrollo social.

En relación con esto, cabe comentar también que la participación en espacios extraescolares, como lo son las actividades comunitarias, contribuyen al desarrollo de otras habilidades en los estudiantes, que se revierten en un mejor desempeño académico, a la vez que son predictoras de mayor éxito en la vida personal y social de los individuos. Esta hipótesis ha sido investigada desde diferentes puntos de vista, por ejemplo, en los trabajos de O'Dea, 1994, Moriana et al., 2006 y Carmona y Sánchez, 2011.

Finalmente, es preciso anotar que todavía quedan puntos pendientes en lo que se refiere a las estrategias y prácticas pedagógicas que propicien condiciones adecuadas para la participación en el desarrollo de las clases. Esto sobrepasa los propósitos y alcances de este estudio que llevaría una investigación diferente ligada al clima democrático en el salón de clase y que precisamente es una de las limitaciones de este artículo, ya que la base estadística del ICCS no ofrece estas posibilidades.

\section{Referencias bibliográficas}

Akbari, R. yAllvar, N. (2010). Teacher characteristics as predictors of students' academic achievement. The Electronic Journal for English as a Second Language, 4, 13.

Alivernini, F. y Manganelli, S. (2011). Is there a relationship between openness in classroom discussion and students' knowledge in civic and citizenship education? Procedia Social and Behavioral Sciences, 15. Recuperado de http://www.sciencedirect.com/science/article/pii/ S1877042811008615

Álvarez, A. (2005). Poder, democratización y formación ciudadana. Cali: Universidad del Valle y Alcaldía de Santiago de Cali.

Álvarez, A. et al. (2002). Gobierno y justicia en la escuela: democracia a medio camino. Cali: Universidad del Valle y Colciencias.

Carmona, C. y Sánchez, P. (2011). Actividades extraescolares y rendimiento académico: dife- 
rencias en autoconcepto y género. Revista de Investigación Educativa, 29(2).

Cox, C., Jaramillo, R. y Reimers, F. (2005). Educar para la ciudadanía y la democracia en las Américas: una agenda para la acción. Nueva York: Banco Interamericano de Desarrollo.

Darling-Hammond, L. (2000). Teacher quality and student achievement: a review of state policy evidence. Education Policy Analysis Archive, 8, 1. Recuperado de http://epaa.asu.edu/ ojs/article/view/392/515

Dewey, J. (1995). Democracia y educación. Madrid: Ediciones Morata.

Durkheim, E. (1996). Educación y sociología. Barcelona: Ediciones Península.

Durkheim, E. (2002). La educación moral. Madrid: Editorial Trotta.

Echavarría, C. (2003). La escuela: un escenario de formación y socialización para la construcción de identidad moral. Revista Latinoamericana de Ciencias Sociales, Niñez y Juventud, 2(1).

García Roca, J. (2007). Educación para la ciudadanía. Recuperado de www.fespinal.com/espinal/llib/es149.pdf

Gil-Villa, F. (1997). La participación democrática en la escuela. De cómo los agentes educativos se las ingenian para gobernarse. Colombia: Cooperativa Editorial Magisterio.

Guttman, A. (2001). La educación democrática. Una teoría política de la educación. España: Paidós.

Instituto Colombiano para la Evaluación de la Educación (ICFES). (2011). Estudio internacional de educación cívica y ciudadana -ICCS 2009. Informe de resultados de Colombia, Bogotá.

León, A. et al. (2012). Tipos de aprendizaje promovidos por los profesores de matemática y ciencias naturales del sector oficial del departamento del Quindío, Colombia. Revista Científica Guillermo de Ockham, 2(10), 49-63.

Losito, B. y Mintrop, H. (2001). The teaching of civic education. En J. Torney-Purta, R. Lehmann, H. Oswald y W. Schulz (ed.), Citizenship and education in twenty-eight countries. Ámsterdam: International Association for the Evaluation of Educational Achievement (IEA).

Mapiasse, S. (2007). Influence of the democratic climate of classrooms on student civic learning in North Sulawesi, Indo- 
nesia. International Education Journal, 8(2), 393-407.

Mayer, D. et al., (2000). Monitoring school quality: an indicators report. Washington: National Center for Education Statistics.

Molina, N. y Pérez, I. (2006). El clima de relaciones interpersonales en el aula. Un caso de estudio. Paradigma, 2, 193-219.

Moriana, J. et al. (2006). Actividades extraescolares y rendimiento académico en alumnos de educación secundaria. Revista Electrónica de Investigación Psicoeducativa, 8(4), 1.

O'Dea, J. (1994). The effect of extracurricular activities on academic achievement. (Thesis for the Degree Specialist of Education). lowa, Drake University, School of Education,

Orellana, C. y Minte, A. (2011). Conocimiento cívico e intención de inscripción electoral de los estudiantes de enseñanza media de la comuna de Concepción, Chile. Horizontes Educacionales, 2(16).

Pinilla, A. y Torres, J. (2006). De la educación para la democracia a la formación ciudadana: una década de incertidumbres. Bogotá: Universidad Pedagógica Nacional.

Ruiz, A. y Chaux, E. (2005). La formación de competencias ciudadanas. Bogotá: Ascofade.

Schulz, W. et al. (2008). Estudio internacional sobre educación cívica y ciudadana. Marco de la evalua- ción. Ámsterdam: Agencia Internacional para la Evaluación del Rendimiento Educativo (IEA).

Schulz, W. et al. (2010). Resultados iniciales del estudio internacional de educación cívica y ciudadana de la IEA. Ámsterdam: Agencia Internacional para la Evaluación del Rendimiento Educativo (IEA).

Torney-Purta, J. et al. (2005a). How Teachers' Preparation relates to Students' Civic Knowledge and Engagement in the United States: Analysis from the IEA Civic Education Study. Nueva York: University Of Maryland.- Recuperado de www. cívicayouth. org/popups/factsheets/FS_ AIE _ Maestro _ preparación.pdf

Torney-Purta, J. et al. (2005b). Teachers' Educational Experience And Confidence In Relation To Students' Civic Knowledge Across Countries. International Journal of Citizenship and Teacher Education, 1, 1. Recuperado de http://www.citized.info

Unesco. (1996). La educación encierra un tesoro. Informe para la Unesco de la Comisión Internacional de Educación para el siglo XXI, presidida por Jacques Delors, Madrid. Autor.

Unesco. (2008). Convivencia democrática, inclusión y cultura de paz. Lecciones desde la práctica educativa innovadora en América Latina. Chile: Autor.

Valencia Serna, A. (2013). La educación para la ciudadanía en 
Colombia: un análisis a partir de las pruebas internacionales sobre educación cívica de la IEA. (Trabajo de grado programa de Maestría en Sociología). Cali, Universidad del Valle.

Valencia Serna, A. y Vivas Pacheco, H. (en prensa). La educación para la ciudadanía en Colombia: la importancia de la apertura para la discusión en el salón de clase.

Vivas Pacheco, H. y Valencia Serna, A. (2011). Logros académiCos, experiencia y prácticas pedagógicas de los docentes en educación para la ciudadanía. Cartagena. Ponencia presentada en el I Congreso Iberoamericano y IV Nacional por una Educación de Calidad. Recuperado de http://www.porunaeducaciondecalidad.org/Congreso/Html/IV\%20Memorias. $\mathrm{html}$ 